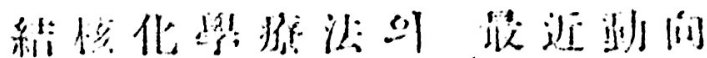

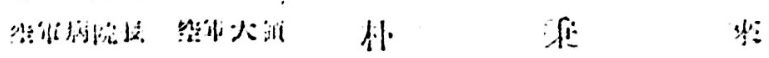

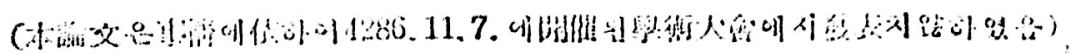

$$
\text { - } 11 \text { : }
$$

(1) $1 \%$ is

(2) 估 1 ! 组

r 1.1 此

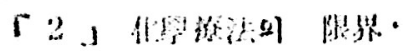

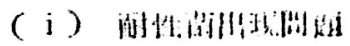

(ii) but

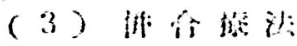

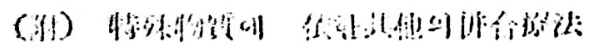

(1) $1 \mathrm{j} \quad \mathrm{i}$

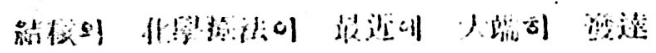

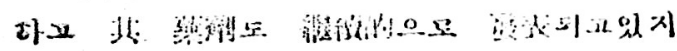

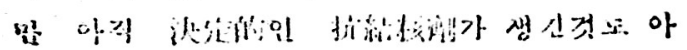

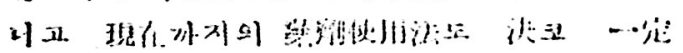

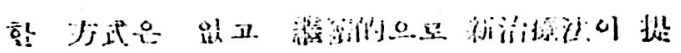

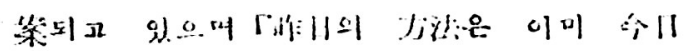

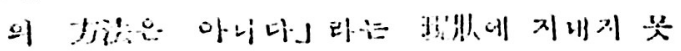
하고 영다

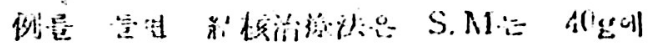

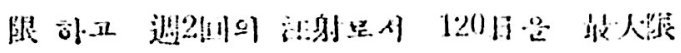

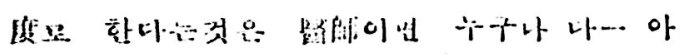

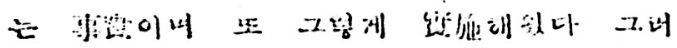

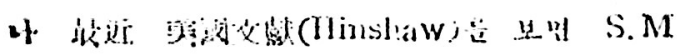

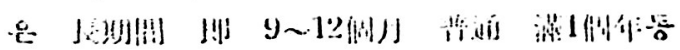

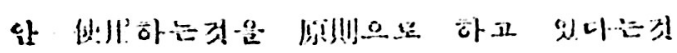
은안 후가였다

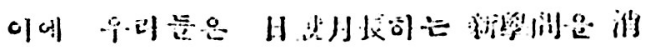

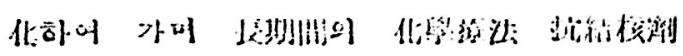

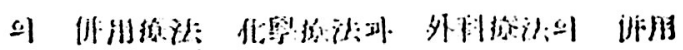

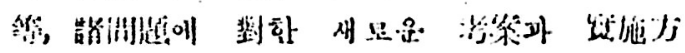

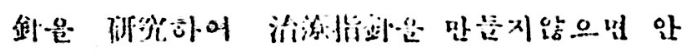
되칬나

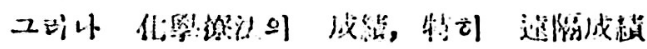

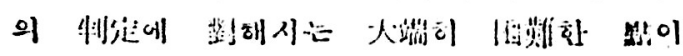

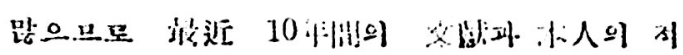

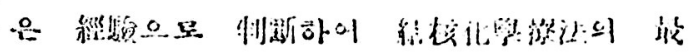

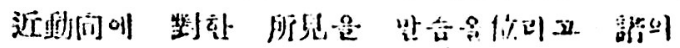

fiont

$$
\text { (2) } 10 \%
$$

$r 1\lrcorner$ W

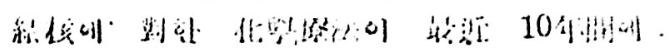

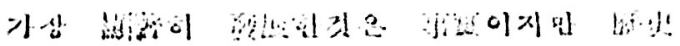

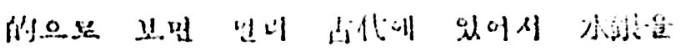

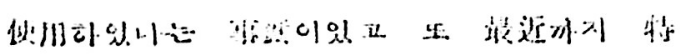

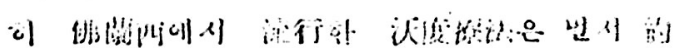

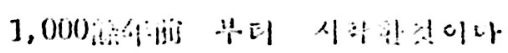

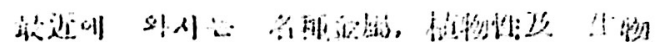

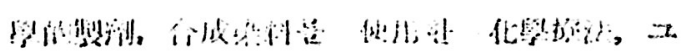

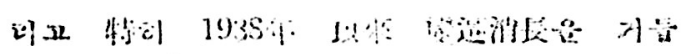

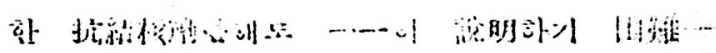

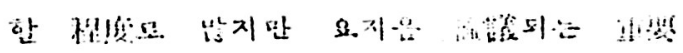

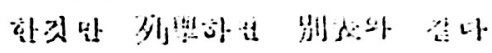

$$
\text { (列 1) }
$$

1. Sulfinamidifin, Sulfadiazone

2. Sulfone $2 \pi$ astitht
a) (incosulfurk Emlitait Promin)
b) Thiazolsulione (Pconizate)

3. Sujplet nne

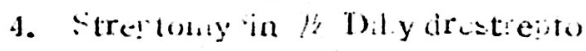
Iii)

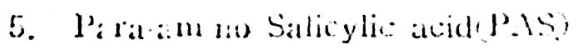

6. Thiosentartane Thl, l698, Thion ne, smit: $0 \%$ onte, conte..ents

7. Nicotinic alid atstint
a) 1:onicutinic Acicl Hycirazicle (INA 11. Rinaifone Isoniazici)

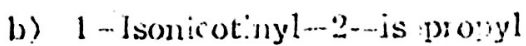
Jyjuracine Marsilid, Iproniazid)
c) Pyrazinamidedoldnamide

8. Nionyzin

11) Aureonycin

10. Terrangtin 12, Mysomycin

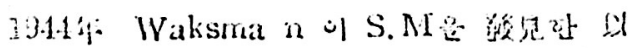




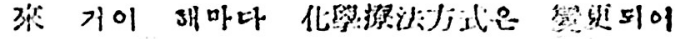

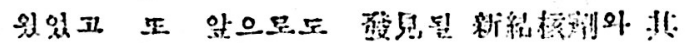

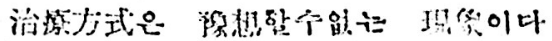

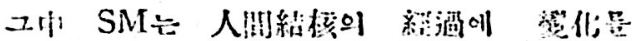

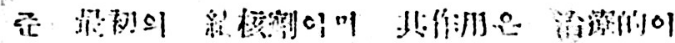

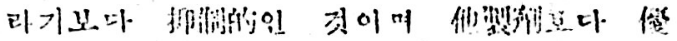

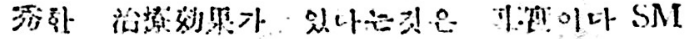

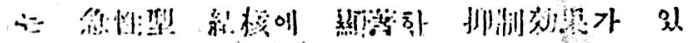

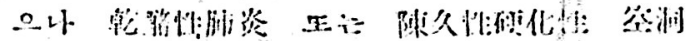

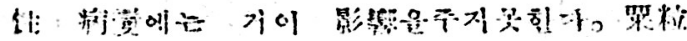

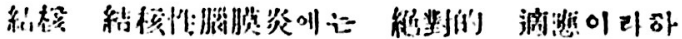

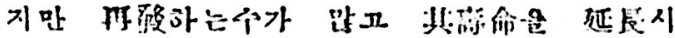

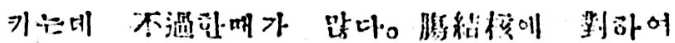

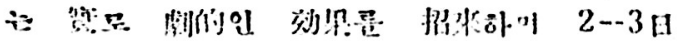

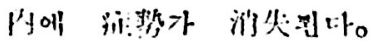

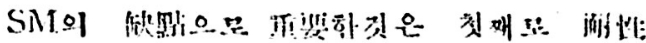

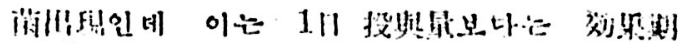

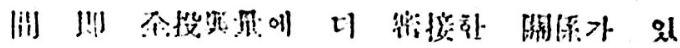

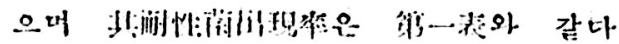
一躬 - 或-

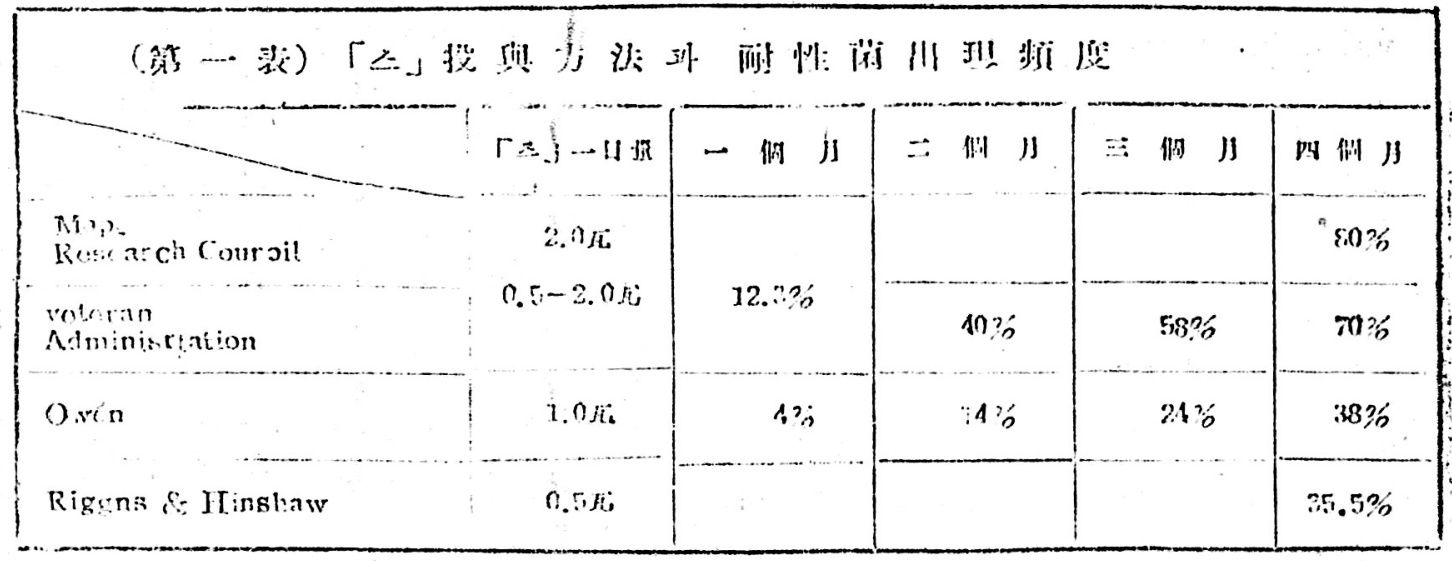

SNA

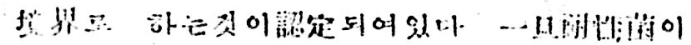
- xy

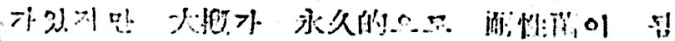

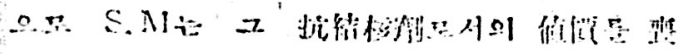

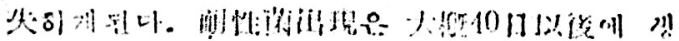

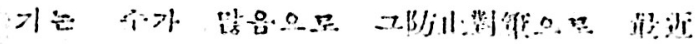

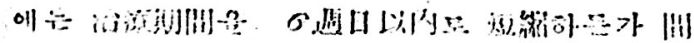

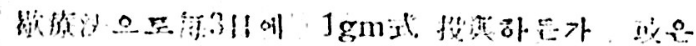

PA.sit Jsoniaz; dy

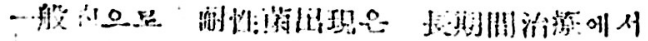

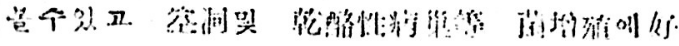

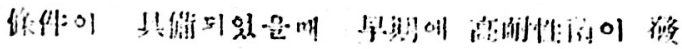

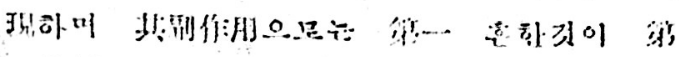

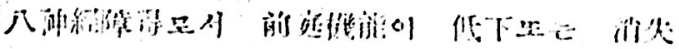

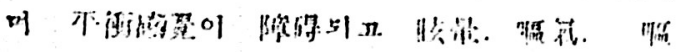

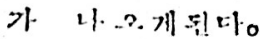

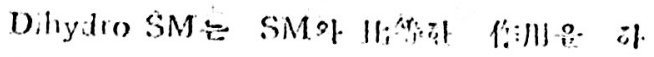

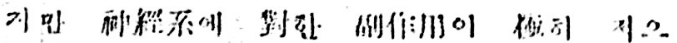

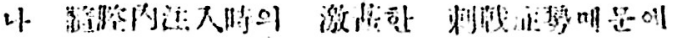

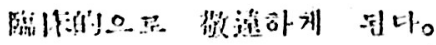

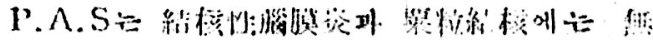

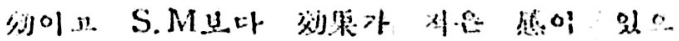

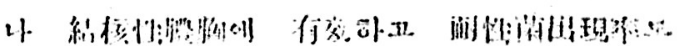

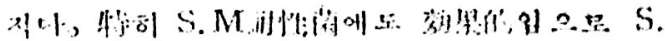

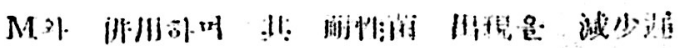

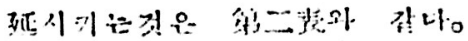

$$
\text { 一射 } 2 \text { 粈- }
$$

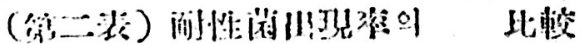

\begin{tabular}{|c|c|c|c|c|}
\hline$f_{1} y_{1} s_{11}$ & 1 & 11 & 111 & IV \\
\hline 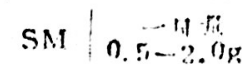 & 12 & 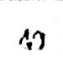 & 55 & $700 \%$ \\
\hline $\begin{array}{c}S M \\
\text { L }\end{array}$ & 0 & 12 & 15 & 80 \\
\hline 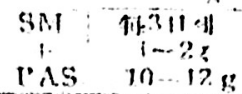 & 0 & 0 & 0 & 0 \\
\hline
\end{tabular}




\section{intio ing}

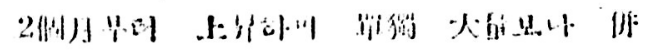

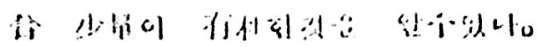

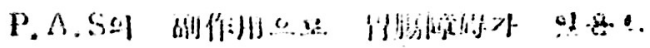

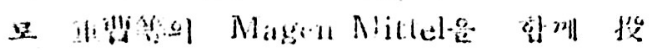
bi) क्षेเी,

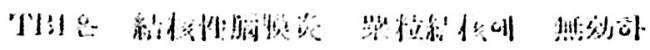

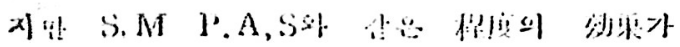
9.

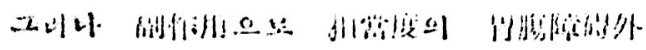

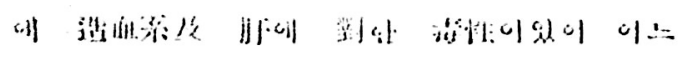

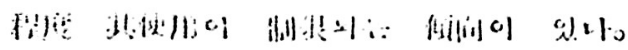

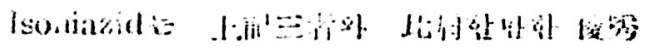

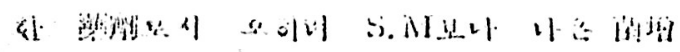

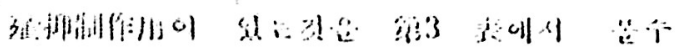
if 4 it.

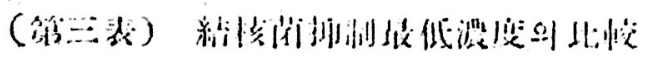
(小湖路)
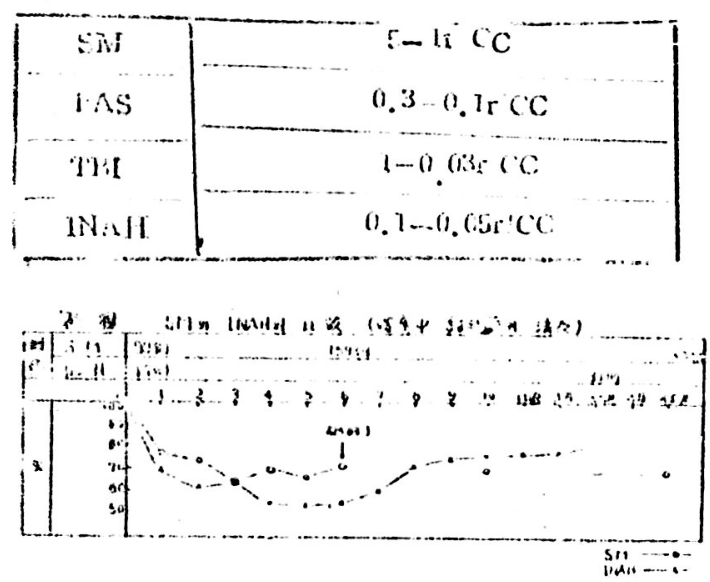

n)3

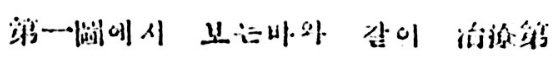

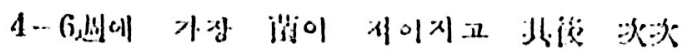

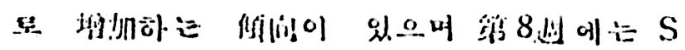

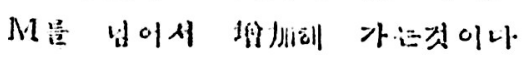

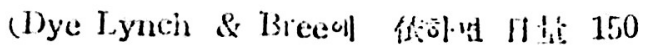

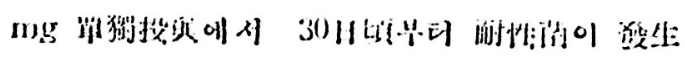

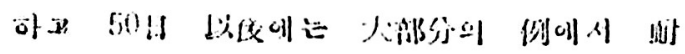

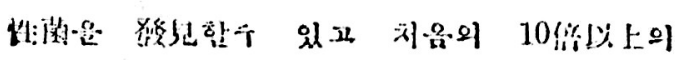

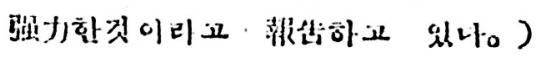

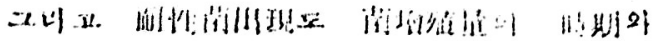

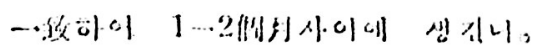

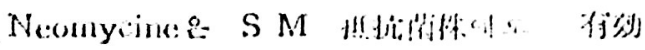

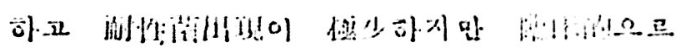

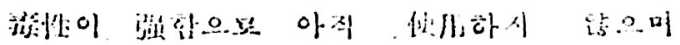

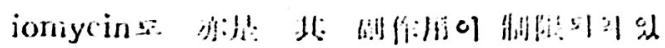
나.

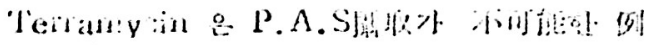

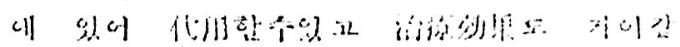

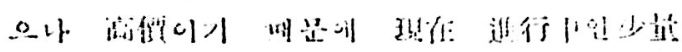

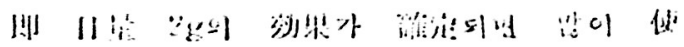

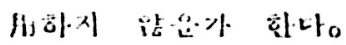

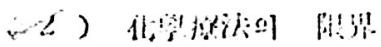

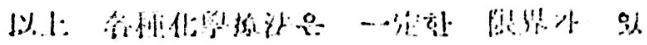

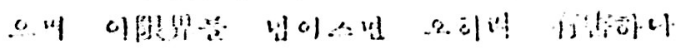

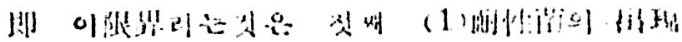

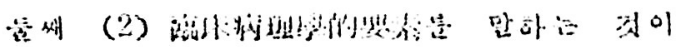
10

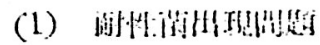

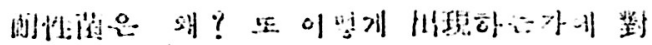

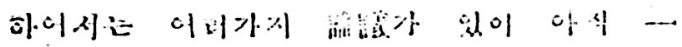

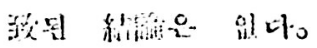

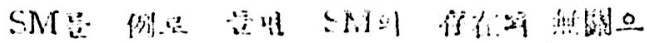

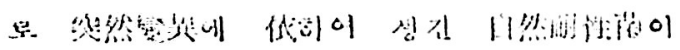

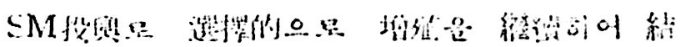
h 任!

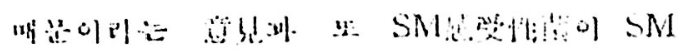
活解性

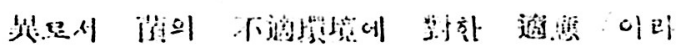

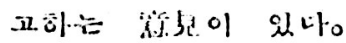

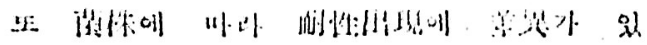
으내 113

1) S.M. Resistant Strain, Meda메시 S

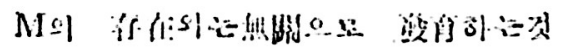

2) S.M, Fnbanced Strain, Mediact 싀 S

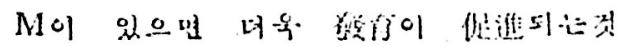
3) S.M, De!erdent Strain S.M. 가있

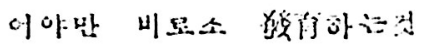

원의 세가지가 있나교 노고욌닝

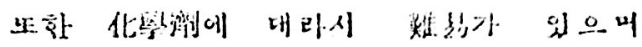

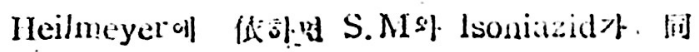




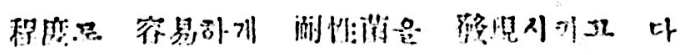
음 P.A.S. 'IJI의 顼으르 되이있으머 TB I이 n-

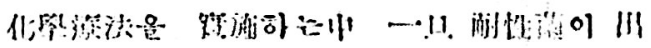

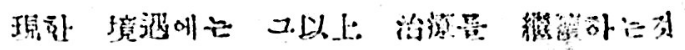

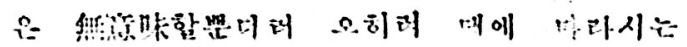

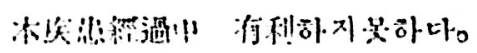

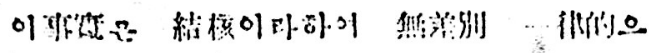

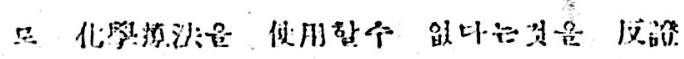
하는칭 이당

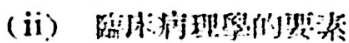

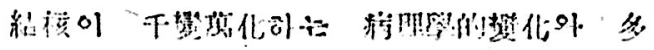

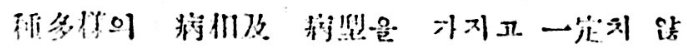

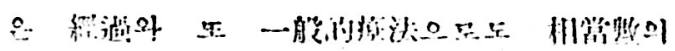

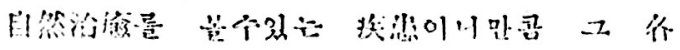

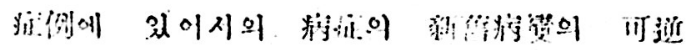

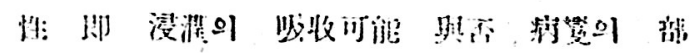

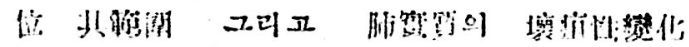

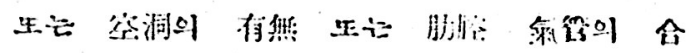

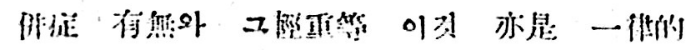

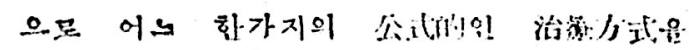

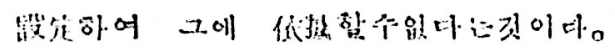

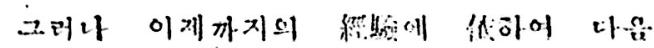

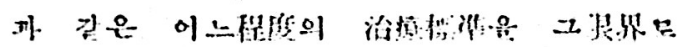

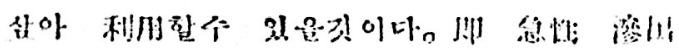

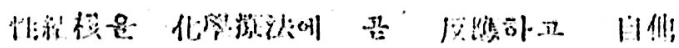

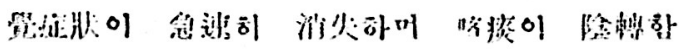

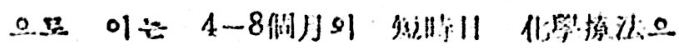

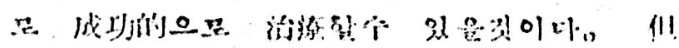

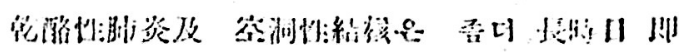

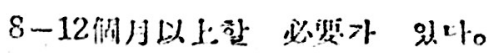

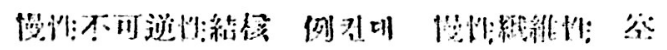

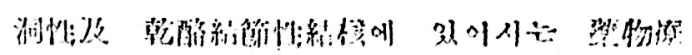

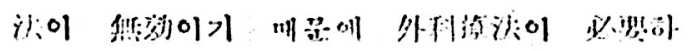

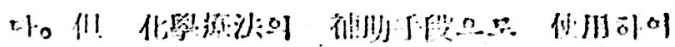

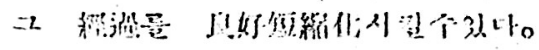

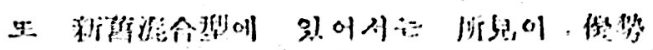

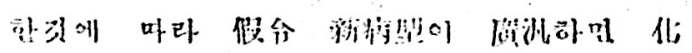

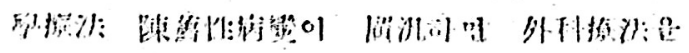

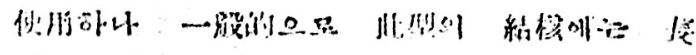

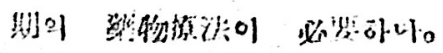

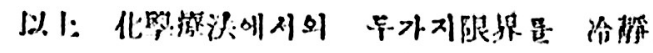

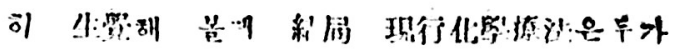

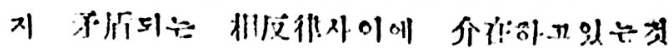
이다 한두일 다응

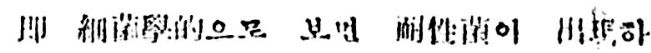

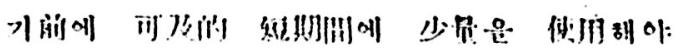

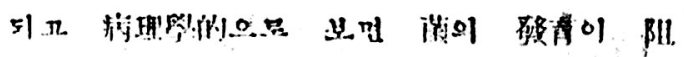

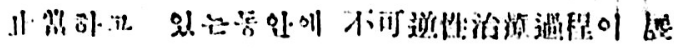

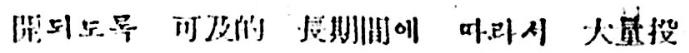

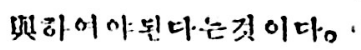

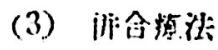

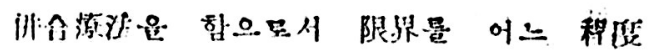

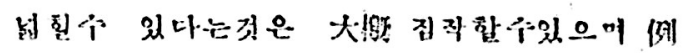

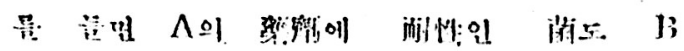

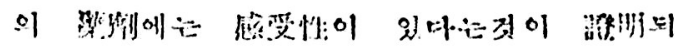

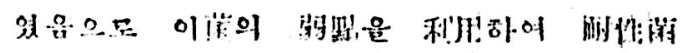

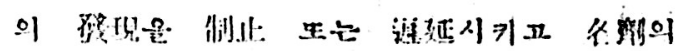

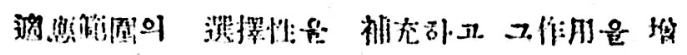

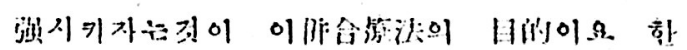

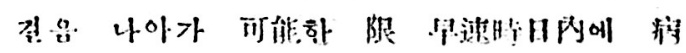

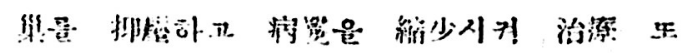
i billy (Good chronic: Case "no

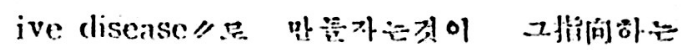
바.이 다,

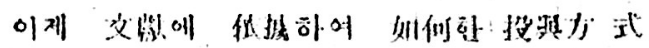

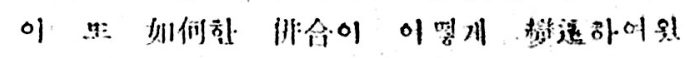

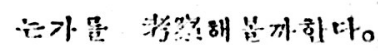

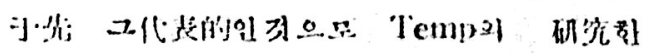

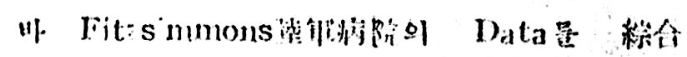

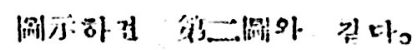

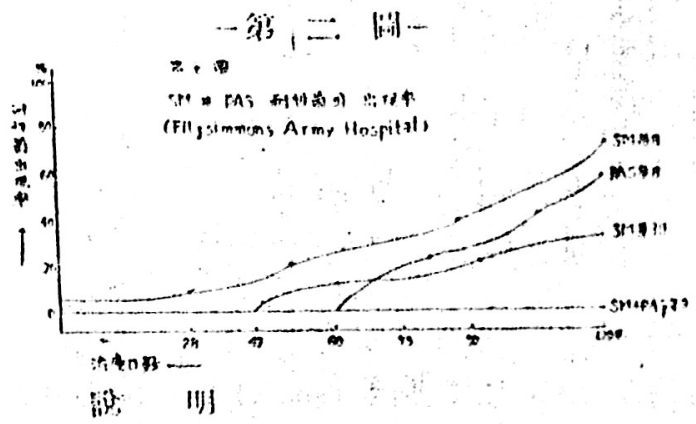

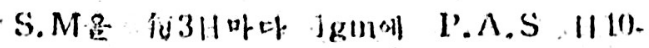

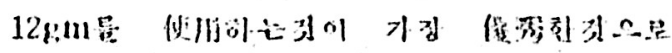




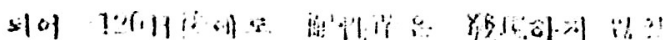
10

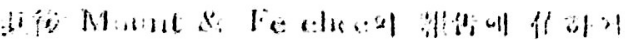

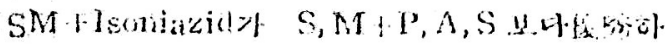

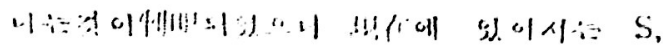

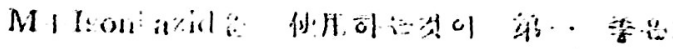

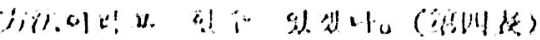

的明

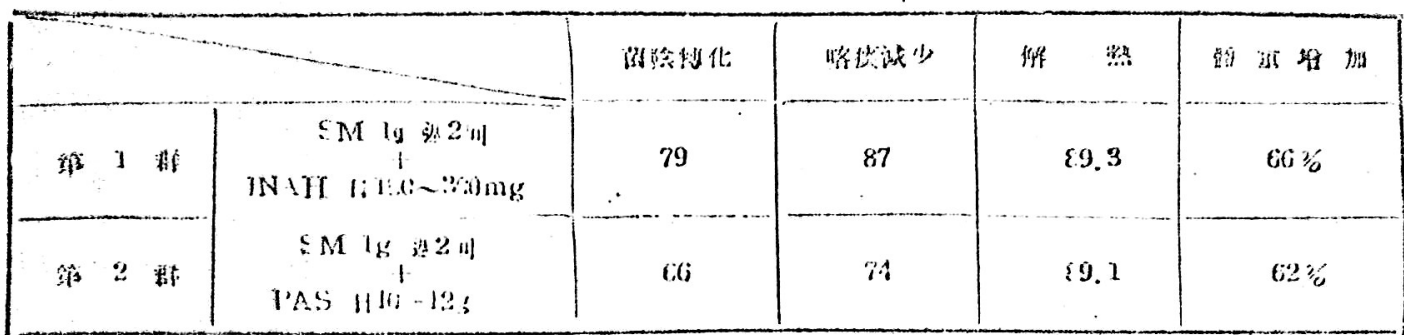

绕 144

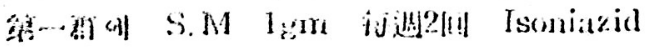

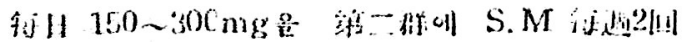

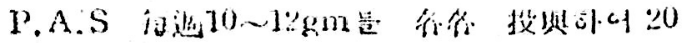

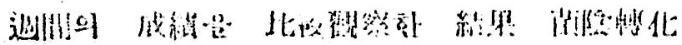

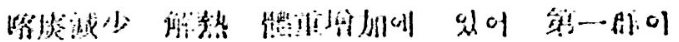

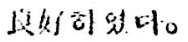

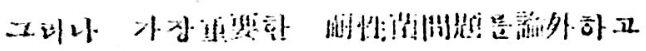

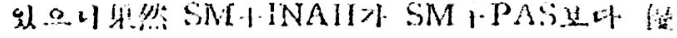

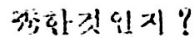

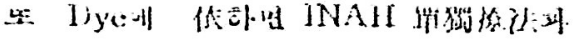

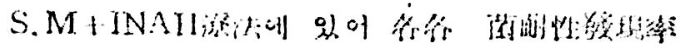

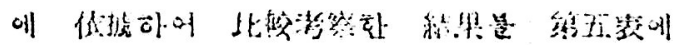

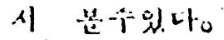

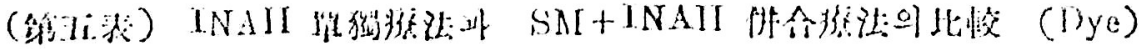

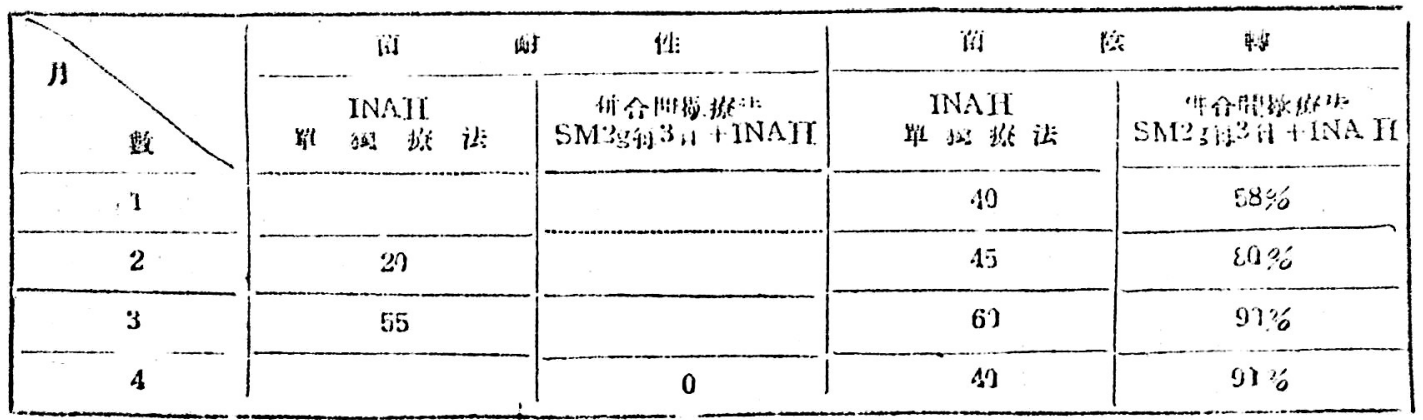

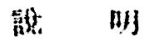

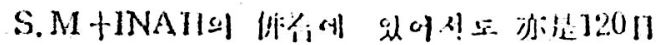

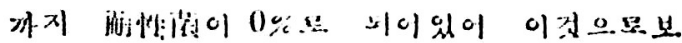

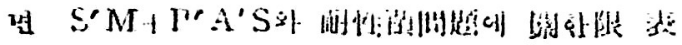

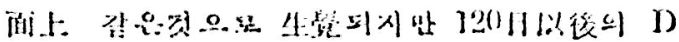

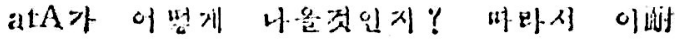

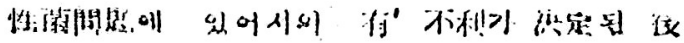

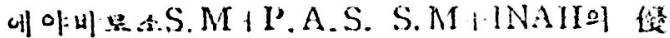

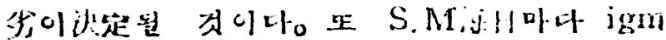

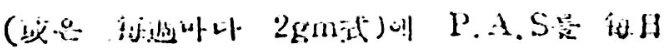

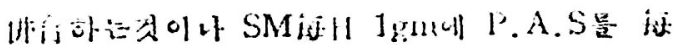

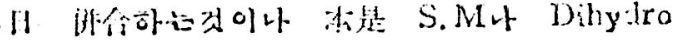

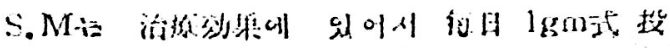




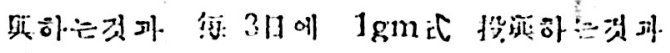

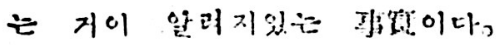

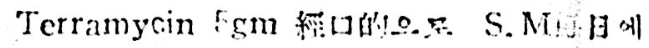

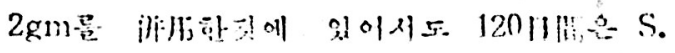

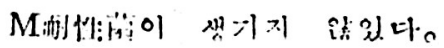

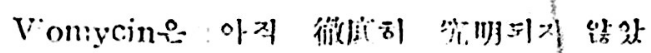

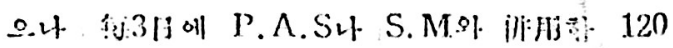

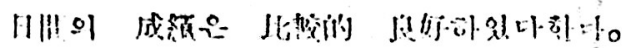

TTI에 있어서 TJ3I 100mg과 S.M $2 \mathrm{gm}$ 何:

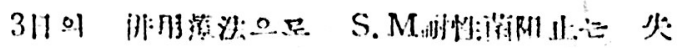

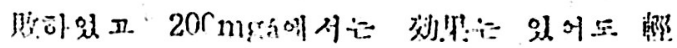

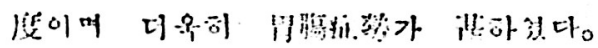

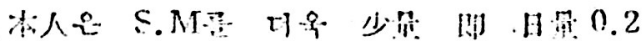

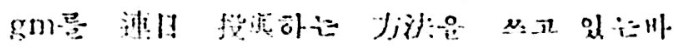

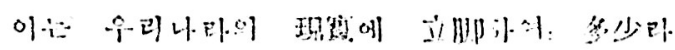

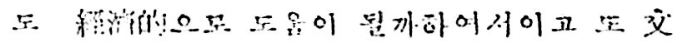

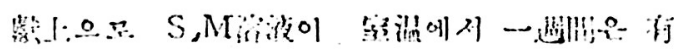

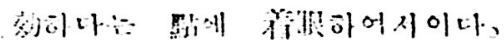

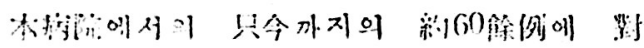

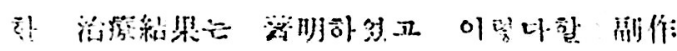
His 도 연 견다,

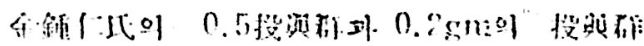

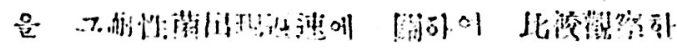

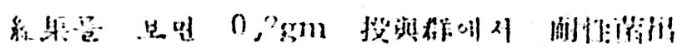

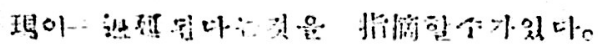

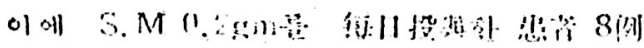

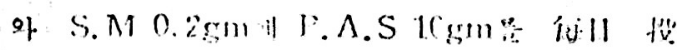

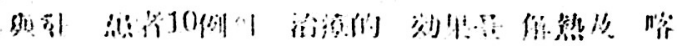

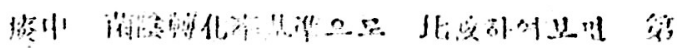

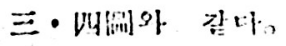

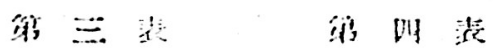

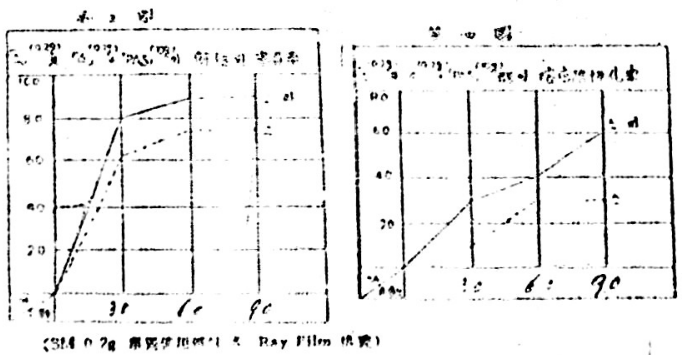

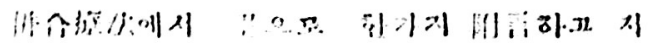

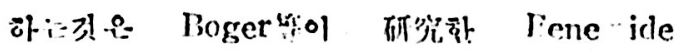

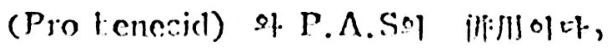

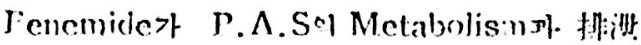

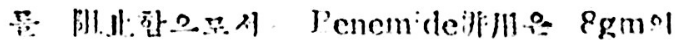

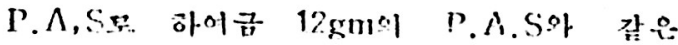

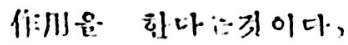

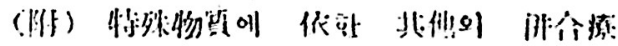

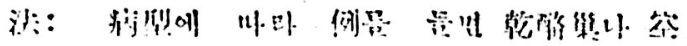

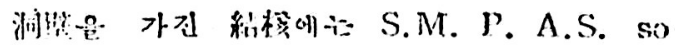

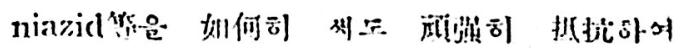

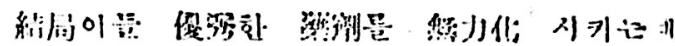
가 니다.

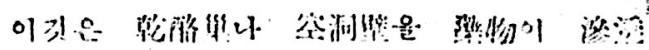

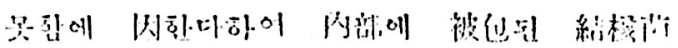

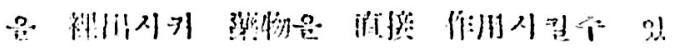

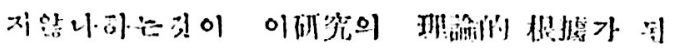
칭이다.

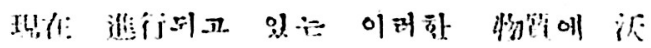
IT.M!!! ( KI) Tulerculin. Strertokinase of

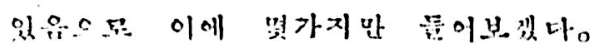

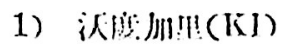

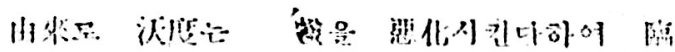

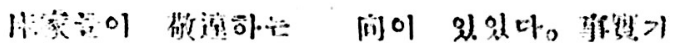

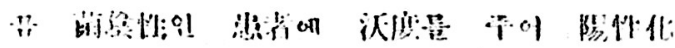

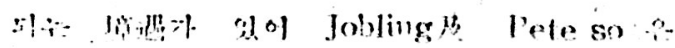

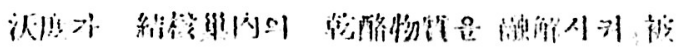

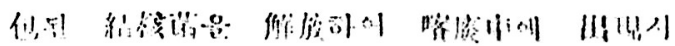

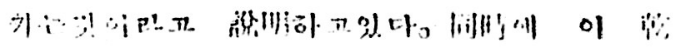

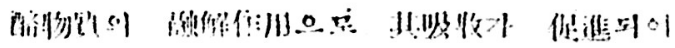

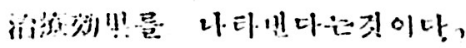

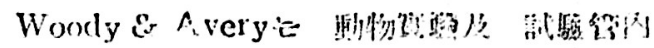

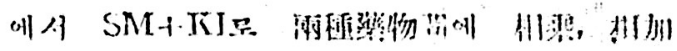

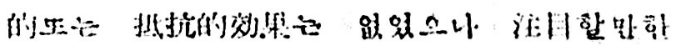

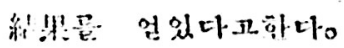

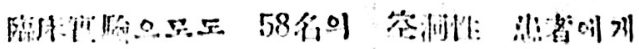

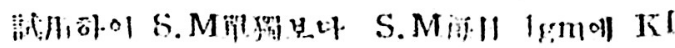

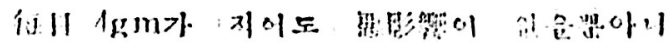

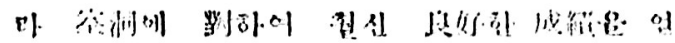

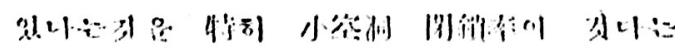

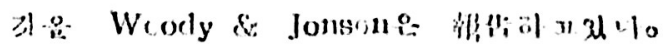




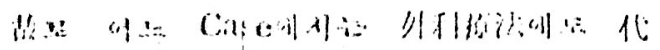

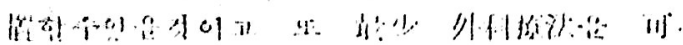

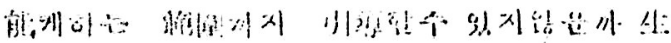

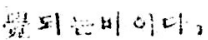

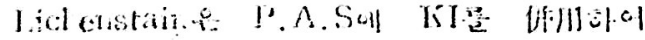

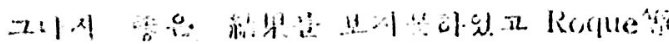

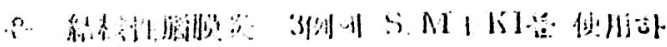

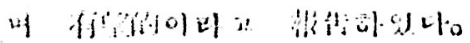

\section{2) Pul trculin}

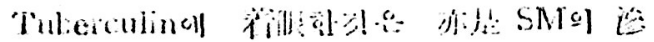

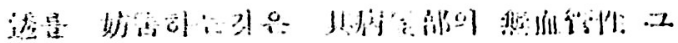

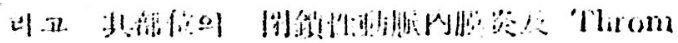

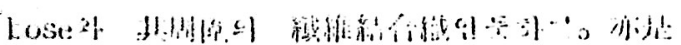

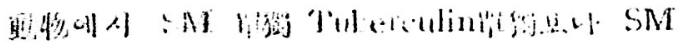

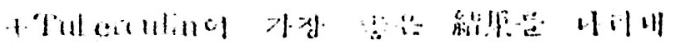
914.

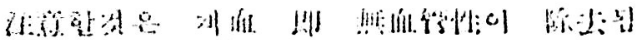

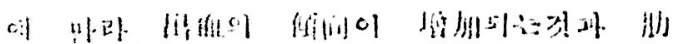

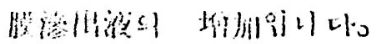

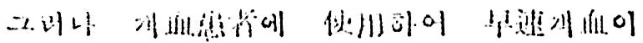

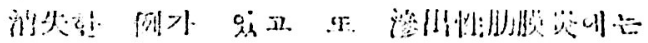

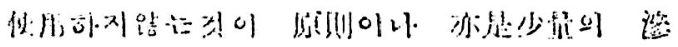

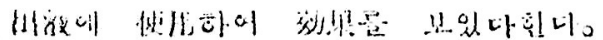

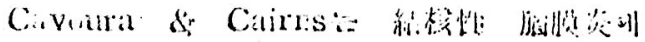

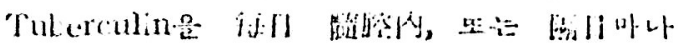

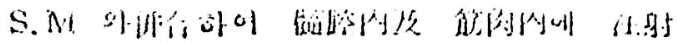

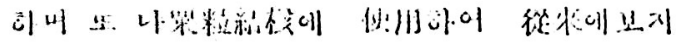

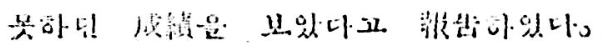

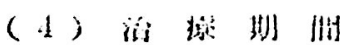

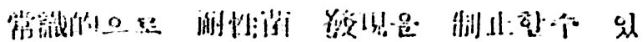

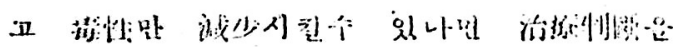

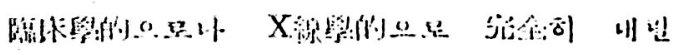

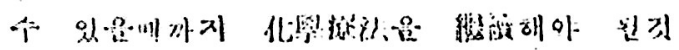

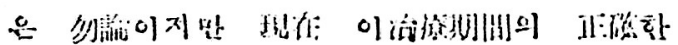

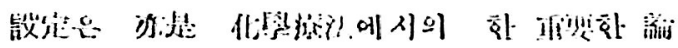

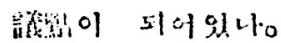

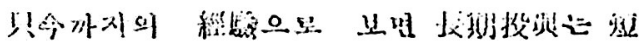

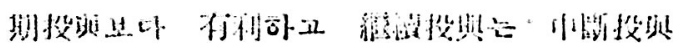

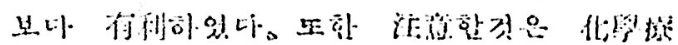

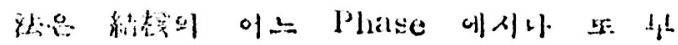

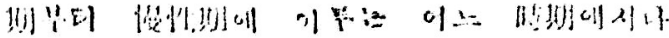

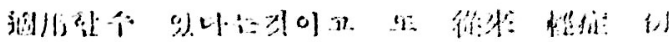

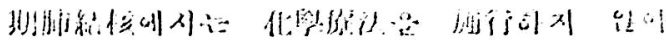

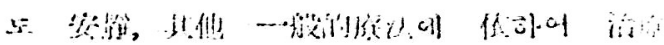

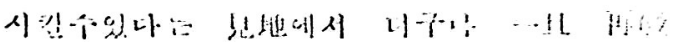

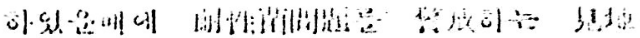

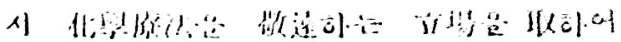

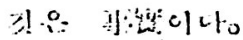

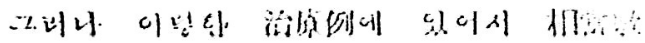

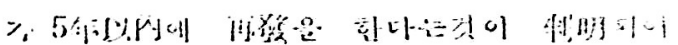

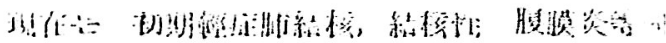

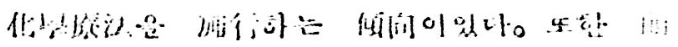

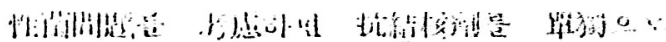

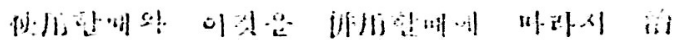

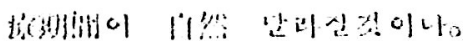

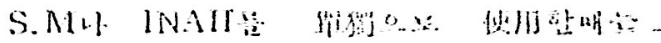

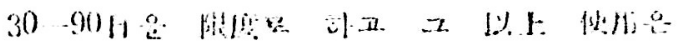

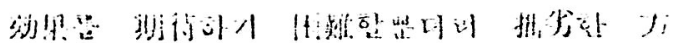

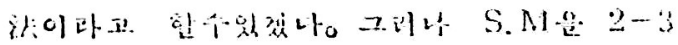

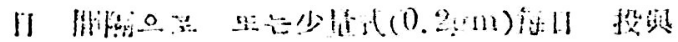

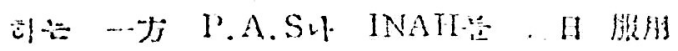

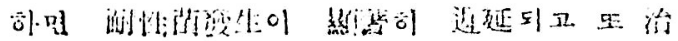

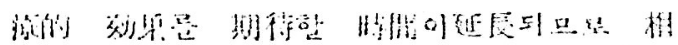

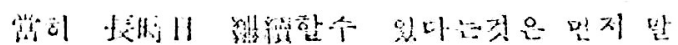
순드린싸워. 갈나잉

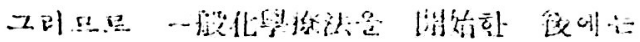

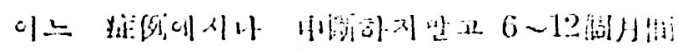

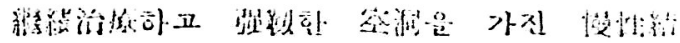

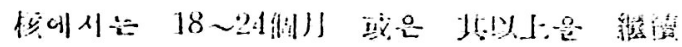
해야 닌경이다.

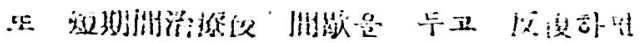

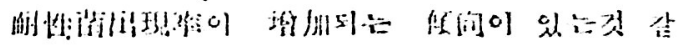

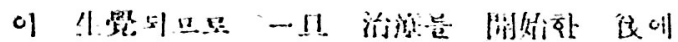

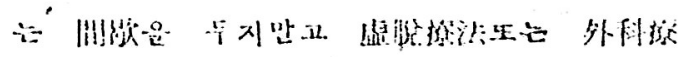

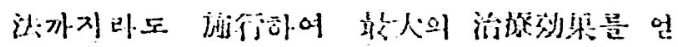

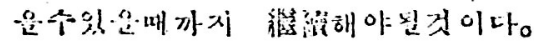

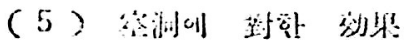

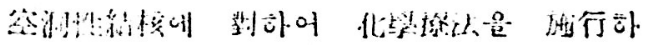

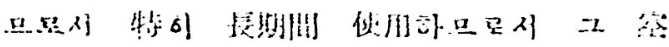

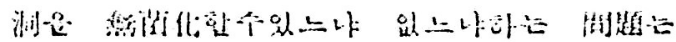




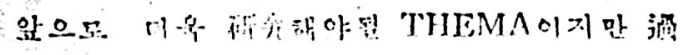

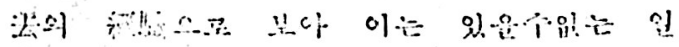
이 리하갰다.

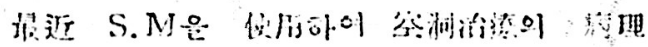

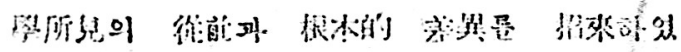

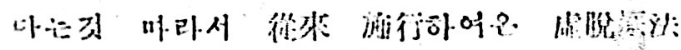

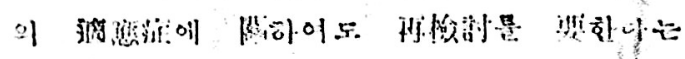
것은 Aure : Back, liatz \& Smaily

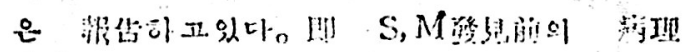

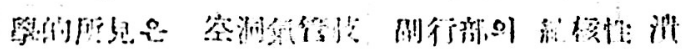
yititiol if

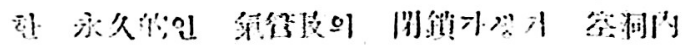

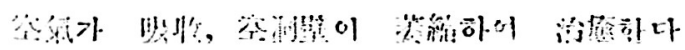

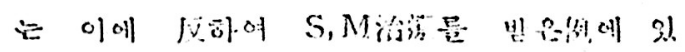

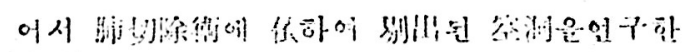

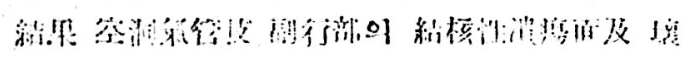

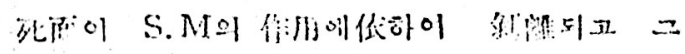

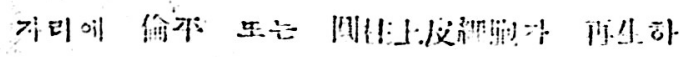

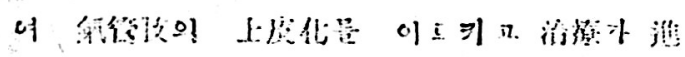

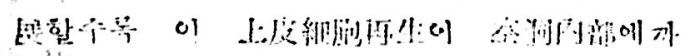

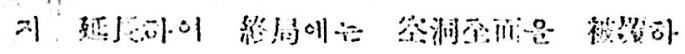

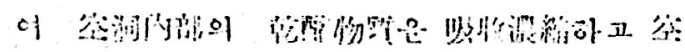

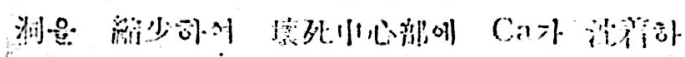

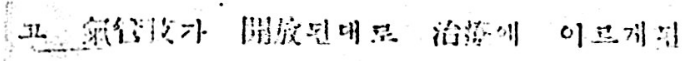

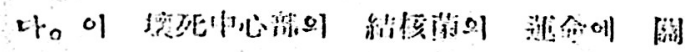

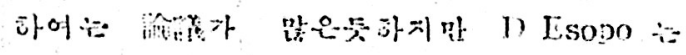

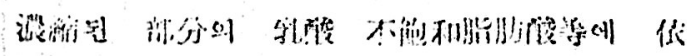

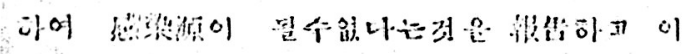

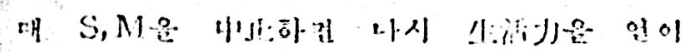

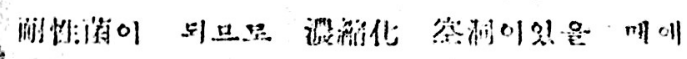

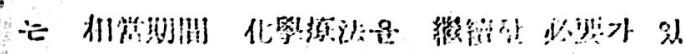
다.

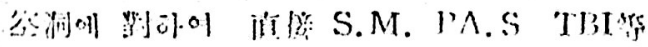

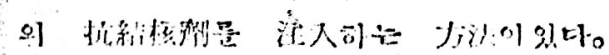

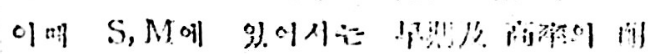

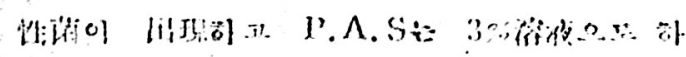
여

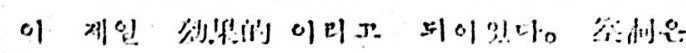

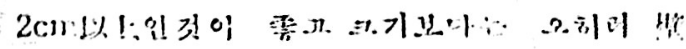

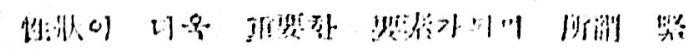

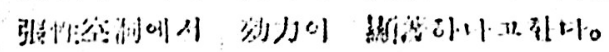

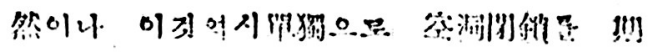

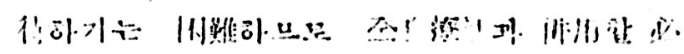

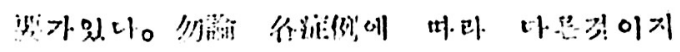

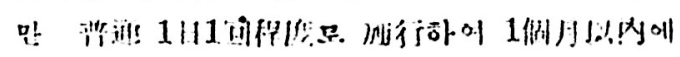

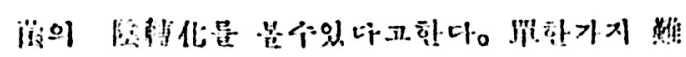

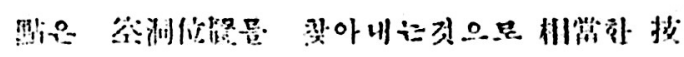

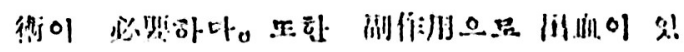

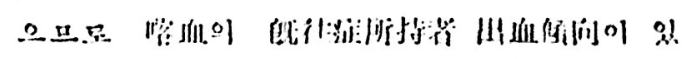

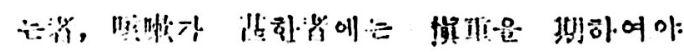

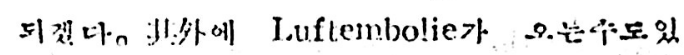
다꼬하ㄴㅏㅏ응

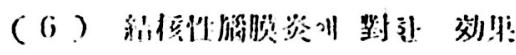

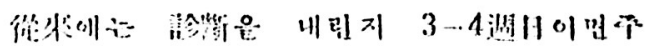

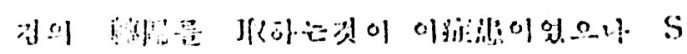

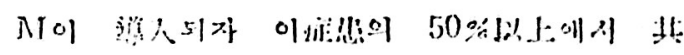

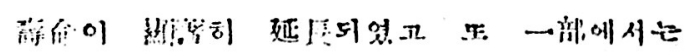

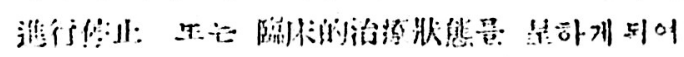
Mtapts Meningitis Tuberculin Chronica " aㅏ

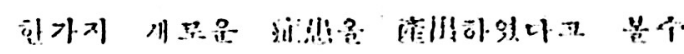

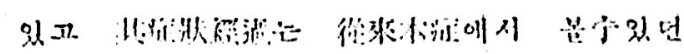

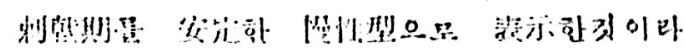

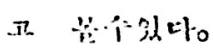

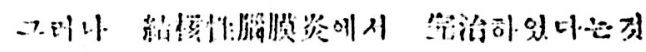

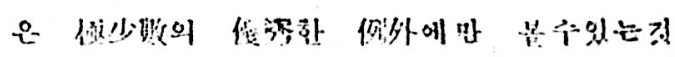

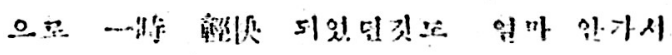

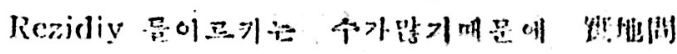

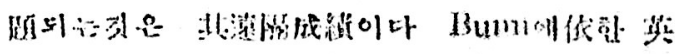

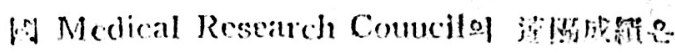

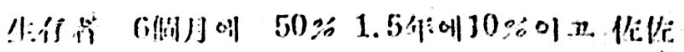

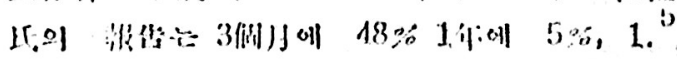

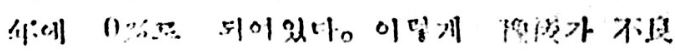

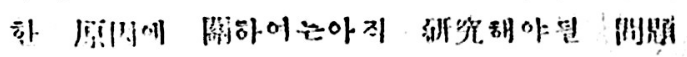

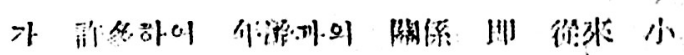

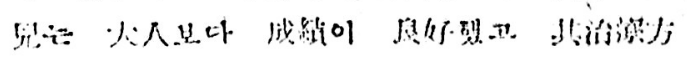
:

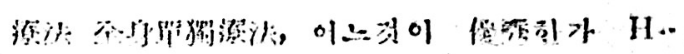
inshaw

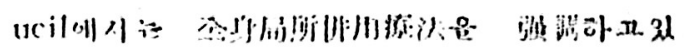
$\therefore$ 메 l.1.

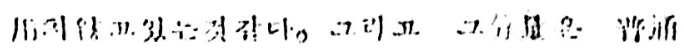

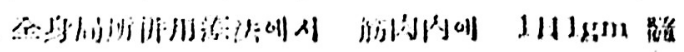





\section{6. 尖 防}

1) T.F.Pain et al; "SM in the treatment of meningitis" 2)W. McDermott et al; "SM in the treatment of Tb. in humane" I. Meningitis ard generalized hematog enous" Tb. 3) C. Muschenhein ct al; "SM in the treatment of Tb. in humans, JI. Pulmonary Tb. 以I: Annals of Int. Med, 1047. $\mathrm{xxx}$ 1.4) iV. Mclermott et al; "Toxicity of SM" 5) II. C. Sweaney;

"SM in tuberculous enteritis." (b)l: C. de Savilsch; "An anti

biotic precursor of SM" 以. $\Lambda \mathrm{m}$. Rev. of Tb. Nov. 19.17. 7) M. W. lisisher et al; "SM and bed-rest in the treatment of lumonary'l'b." $\Lambda$ n. Rev. of 'Th. Dec. 3947. 8) Jiolmer Jone $\Lambda$. ; "The Chemotherany of pulmonary "Tb" 9)H. $M$. Riggins et al; "Antibiotic ard chemotherapy of Tb" i0)IT. W. Fisther; "SM in resistant bacilli; "Their development during SM therapy of nulmonary 'Tb" 11) Wm. II. Fedelman et al; "SM resistant tubercle bacilli; Lffect of resistance on therapeutic results in tuberculous guinea pigs" S.L. Am. Rev, of Tb. 1918. IVii. 12) D. T. Carr et al; "Nourotoxic reactions to bihydrostrentomycin" IАMA. 143; 14 Aug. 51950 13) Karlson $\Lambda$. G. et al. "The effect of neomycin on Tis. in guinca piss infected with SM-resistant Tb. bacilli" 14) Fordham G. F. ot al; "Tinotional factors in pulmonary Tb" 以.. Am. Rev of Tis. Oct. 1950. 15) I,incoln N. S. et al; "Dihydrostrept!nycin in nulmonary Tb" $\Lambda \mathrm{m}$, Rov, of $\mathrm{Tb}$. 62 ; 6 Dec. 1950. 16) Duncan G. G. et al ; "Neomycin ; results of clinical use in 1.0 cases" JAMA. 145; 2 13. Jan. 1951. 17) Finlaly $\Lambda$. C. et al; "Viomycir: ; a new autibiotic active nerainst Mycobacteria" 18) Bartz Q. R. et al "Vionycin a new tuberculostaic antibiotic" 19)Netzer $S$;"l'en years of pneumoperitoneum" W... Am. Rev. of Tb. 633 ; 1. Jan. 1951. 20) 'Temmel C. W. et al. "Combined intermittent regrimens cmploying $S M$ and $l ' \Lambda S$ in the treatment of pulmomary $T b$ ; a comparison with daily and internittent dosane schedules. $\Lambda$. Rev. of Th. 63;3 Mar, 1951. 21)Hobby (i. J. et al; "The tuberculostatic activity of Teramycin" 22) Bogen 1 . et al ; Chomotherany for all active 1b. W.1. Am. Rev. of Tb. 63 ; 4. Apr. 1951. 23) Domovick R. et al ; "Chemetheraluy of Th. ; iv. Studies with Sulfonesin the mouse" 24. "Current status of antinicr obialtherapy in 'T1," ; Report of clinical subcommitlece of commitles (n) medicel research and lierany of $\Lambda$ mesican Trueleau Society j. l:. $\Lambda$ m. kev. of Tb. 63 ; 5, May. 1951 25) Jichenstein M. $\mathrm{R}$; "Totassium Iotide and

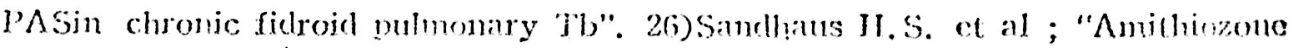
treatment of Pulmonary Th; $\Lambda$ pilot stucy of 21 Jatients" $N \mathrm{I}$. $\Lambda \mathrm{m}$. R.v. of 'Tb. "Chomotherany of Th. in mat" IAMA, 147;3, 15. Sont. 19:1. 28) Aquilina, I. T.

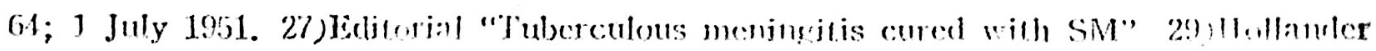

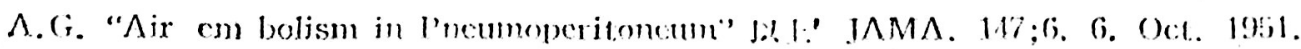

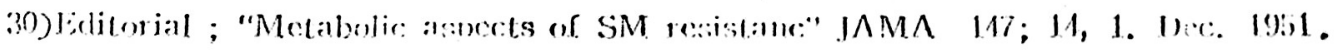

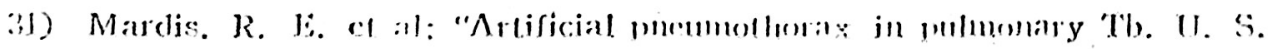
Armed Forces, Med. Jumm. 2; 12, Jec, 19.51. 32) Jacolss l.. C. et al ; "Combined 
SM tuberjulu that!y in pulmonary Th", I. S. Armed lioseces, Med. Journ. 3;

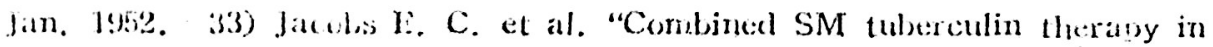

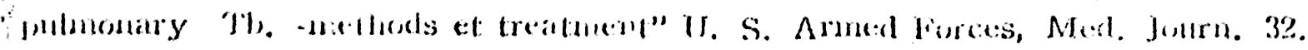

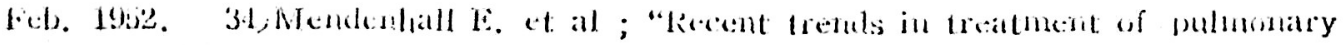
11)". JAMA. 113 ; 8. 23. Feb. 195\%. 35) Solswarte Win. S. ; "Recent drus

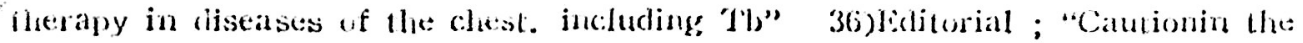
chenditheral,y of Th". 以1.JAMA, 119, 13, 25, July. 19.3. 37)lfamilton M, A, et al ; “lle use of potassium lodiks in Combination with SM in the treatmenta! $\mathrm{Tb}$ in guinea pigs" 38) Bodrowitz, 1, D, et al ; "Gastrointestinal Changes in pheumoleritoneum" 39)Correspondence ; "effect of Iodine on Tb". 以 L. Am. Rev. of Tb. $66 ; 6$ lee 19.2 40) Auerbach O. et al ; "The effect ci SM theran cn the bronchocavitary jurction and its relation to cavity healing". 41) Baces, J. M. et al "The afeot of Corticotronin (ACTH), Dihydro Streptomycin, and Corticotropin-dihyciroystrotomycin on experimental bovite $T$ b in the rablet.

42) Selikoff I.J. et al ; Wilhcirawal Syntptoms unon aiscontinuance of lisoniazi , and Isoniazid thera!y" 43; "Thk curent status of Isoniazid in the treatment of Th ; A. Renort of the Committe on therany" An!. Rev of Tb. $67 ; 2$. Fet. 1453. 41) Machithess, $G, 13$, et al. "Thte batetericilal action of Isoniazid, SN. and Teramycin on extracellu!ar and Intracellubr "l'ubercle Bacilli" 45) Yale 11 .

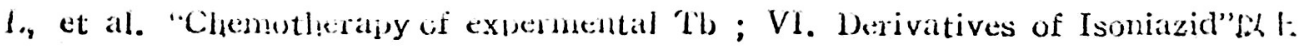
An. Rev. of Th. $67 ; 3$, Mar ; 1953.

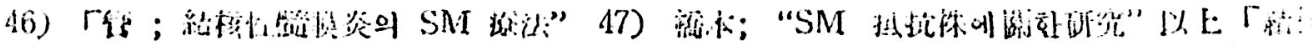
$26 ; 5,1951 ; 5.48$ ) Xill - IL

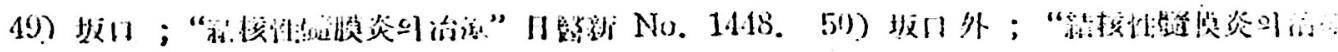

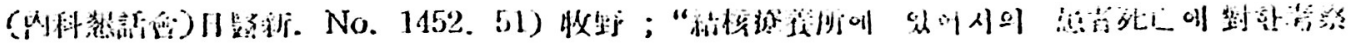

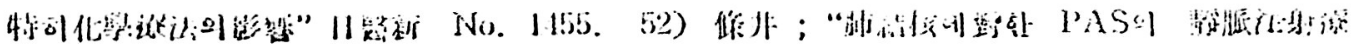

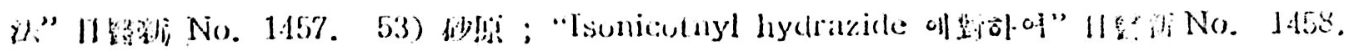

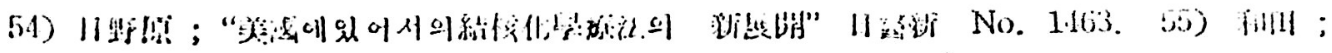

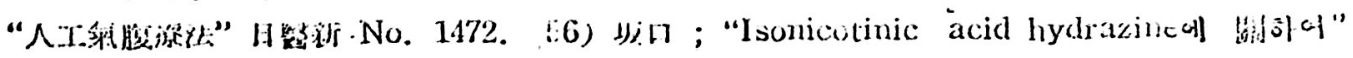

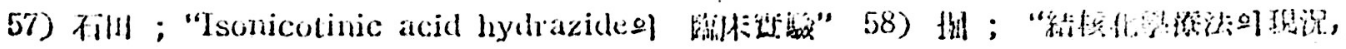

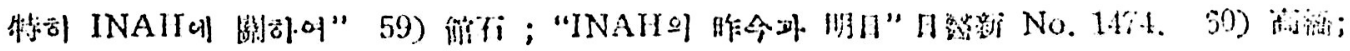

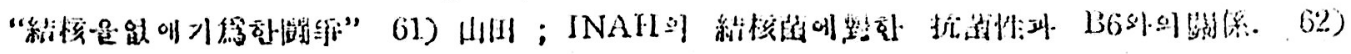

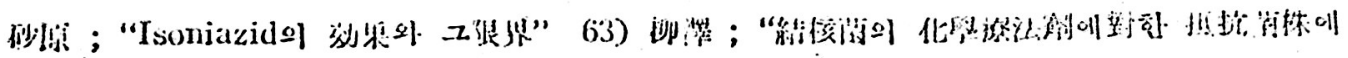

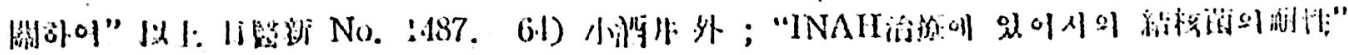

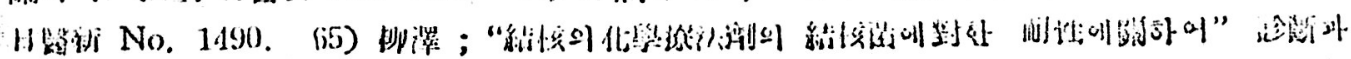
治识 41 ; 3. Nar. 1953. 以上 